\title{
Metodologia de Classificação de Descargas Parciais em Hidrogeradores Utilizando Mapas Auto-organizáveis de Kohonen
}

\author{
Filipe C Fernandes ${ }^{1}$, Rodrigo M. S. Oliveira ${ }^{1}$, Anderson J. C. Sena ${ }^{1}$, \\ Ramon C. F. Araújo ${ }^{1}$, Fabrício J. B. Brito ${ }^{1}$
}

${ }^{1}$ Programa de Pós-Graduação em Engenharia Elétrica - Universidade Federal do Pará

\begin{abstract}
The most used way to evaluate the stator insulation condition in hydrogenerators is partial discharge (PD) monitoring. In this work, a PD classification system is developed using Kohonen self-organizing maps (SOM). Several literature techniques were combined for preprocessing and pattern visualization. It is proposed a methodology that obtains the separation boundaries in the Kohonen map that maximizes the accuracy, besides automating the classification of unknown patterns. The trained Kohonen map presented a high success rate, generalizing the problem to the point of showing subgroups associated to variations of the patterns of the same type of $P D$.
\end{abstract}

Resumo. A maneira mais utilizada para avaliação da condição da isolação estatórica em hidrogeradores é o monitoramento de descargas parciais (DPs). Neste trabalho, é apresentado um sistema de classificação de padrões de DPs usando mapas auto-organizáveis de Kohonen (SOM). Foram combinadas diversas técnicas da literatura para pré-processamento e visualização dos padrões. Propõe-se uma metodologia que obtém as fronteiras de separação no mapa Kohonen que maximizam a acurácia, além de automatizar a classificação de padrões desconhecidos. O mapa de Kohonen treinado apresentou alta taxa de acerto, generalizando o problema a ponto de evidenciar subgrupos associados a variações dos padrões de um mesmo tipo de DP.

\section{Introdução}

O acompanhamento dos níveis de descargas parciais (DPs) é uma das formas mais efetivas de se verificar a condição de equipamento de alta tensão [Stone 2005, Stone 2012]. O monitoramento de DPs geralmente é realizado com a máquina em funcionamento pleno (online) [Stone 2012]. Entretanto, nessas condições os equipamentos estão imersos em um ambiente de intensos níveis de ruídos e interferências, dificultando a medição confiável de sinais DP [Stone 2005].

Por outro lado, quando o equipamento é monitorado continuamente, o grande volume de informações geradas inviabiliza a análise manual dos dados [Oliveira et al. 2017]. Por consequência, faz-se necessário o desenvolvimento de um sistema automático, capaz de filtrar os sinais medidos e classificá-los em termos de tipos de defeito normatizados [IEC 2012], associados à isolação.

A maior parte dos trabalhos endereça o problema utilizando redes neurais [Oliveira et al. 2017] para classificação automática. O mapa auto-organizável de Kohonen, embora pouco utilizado, apresenta as seguintes vantagens:(i) maior interpretabilidade dos resultados, devido à verificação visual do mapa SOM; (ii) necessidade de menos 
amostras para um aprendizado representativo do problema; e (iii) melhor análise de padrões de teste duvidosos, baseada na comparação dos mesmos com as amostras de treino mais próximas no mapa.

Neste contexto, é proposto no presente artigo, um método de classificação de DPs utilizando o mapa de Kohonen. Foram combinadas diversas metodologias encontradas na literatura para filtragem de ruídos, extração de características (features) e visualização dos padrões no mapa SOM. Os dados são pré-processados em duas etapas. Na primeira, os sinais medidos no domínio do tempo são decompostos em várias sub-bandas por transformada wavelet [Zampolo et al. 2015]. Redes neurais são empregadas para identificar as sub-bandas que contêm a parcela mais relevante do sinal de DP, eliminando os ruídos [Zampolo et al. 2015]. Na segunda etapa, são extraídas dos sinais filtrados as features propostas por [Oliveira et al. 2017], as quais são fornecidas como entradas para o treinamento da rede SOM. Após o treino, a técnica de [Han and Song 2003] é empregada para visualizar as regiões do mapa SOM referentes a cada tipo de DP. A contribuição deste trabalho é propor a utilização do mapa SOM com a abordagem supervisionada, em que os rótulos (tipo de DP verdadeiro) dos padrões são definidos a priori por especialista. Com base nesses rótulos, foi desenvolvida uma metodologia que auxilia o especialista a definir as fronteiras de separação ótimas no mapa SOM.

\section{Base de Dados}

A base de dados utilizada neste trabalho é formada inteiramente por 568 amostras medidas de forma online em hidrogeradores da Usina Hidroelétrica de Tucuruí-PA. O osciloscópio utilizado para a aquisição dos dados é o PXI 5133. Uma característica de medições in loco é a baixa relação sinal-ruído, que dificulta a tarefa de classificação das amostras em padrões de defeito associado à DP. Portanto, para uma medição mais confiável, a metodologia de filtragem proposta por [Zampolo et al. 2015] foi utilizada para remover o máximo possível de ruídos e interferências, minimizando a distorção do sinal de interesse.

Ao todo, são consideradas cinco classes de padrões de DPs: cavidade interna, ranhura, corona, superficial e lacuna. Vale ressaltar que os tipos de DP ranhura e corona foram mesclados para formar uma única classe.

\section{Metodologia de Visualização da rede SOM}

Na literatura, há várias metodologias para visualização dos grupos de neurônios (clusters) no mapa SOM. A técnica convencional consiste inicialmente em calcular a média das distâncias dos pesos sinápticos de cada neurônio para os seus vizinhos imediatos (primeira vizinhança, Figura 1). Tais distâncias são codificadas em escala de cores e sobrepostas ao mapa SOM. Entretanto, essa técnica não ressalta as bordas dos clusters de forma eficaz.

O algoritmo de visualização proposto em [Han and Song 2003] foi utilizado neste trabalho em razão de melhor realçar as fronteiras entre os grupos formados. Nesta técnica, distâncias são calculadas para cada neurônio, codificadas em escala de cores cinza (branco corresponde à menor distância, e preto à maior) e sobrepostas ao mapa SOM. A cor de menor distância (branco) é atribuída a todos os neurônios excitados durante o treinamento. Em seguida, para cada neurônio não excitado, calcula-se a distância do mesmo para dois dos neurônios excitados vizinhos de acordo com o algoritmo abaixo: 
1. Iniciando da primeira vizinhança (camada), buscar pelos neurônios excitados camada a camada até que pelo menos dois sejam encontrados no total.

2. Se os neurônios excitados estiverem em camadas diferentes, calcular a distância $d_{i}$ (Equação 1) do neurônio de menor camada em relação a cada um dos neurônios das demais camadas. Caso todos os neurônios estejam em uma mesma camada, calcular as distâncias $d_{i}$ entre todas as combinações dois a dois destes neurônios.

3. Apenas a maior distância calculada no passo 2 é mapeada na escala de cores cinza e sobreposta no neurônio não excitado em questão.

As distâncias $d_{i}$ mencionadas no passo 2 são calculadas através da Equação

$$
d_{i}=\left(\left\|m_{i}-m_{1}\right\|+\left\|m_{i}-m_{2}\right\|\right) \times\left(\frac{\frac{1}{2}+\min \left(\left|a_{1}-a_{2}\right|, 6-\left|a_{1}-a_{2}\right|\right)}{6}\right),
$$

em que $m_{i}, m_{1}$ e $m_{2}$ são os vetores de pesos sinápticos do neurônio não excitado $i$, e de dois neurônios excitados vizinhos quaisquer 1 e 2, respectivamente; $a_{1}$ e $a_{2}$ são os parâmetros angulares dos neurônios 1 e 2. Para obtenção destes parâmetros, a região em torno de $i$ é angularmente dividida em seis zonas iguais. Tais zonas são numeradas de 1 a 6 em sentido anti-horário, conforme Figura 1. O parâmetro angular de um dado neurônio é igual à numeração da zona em que o mesmo está situado.

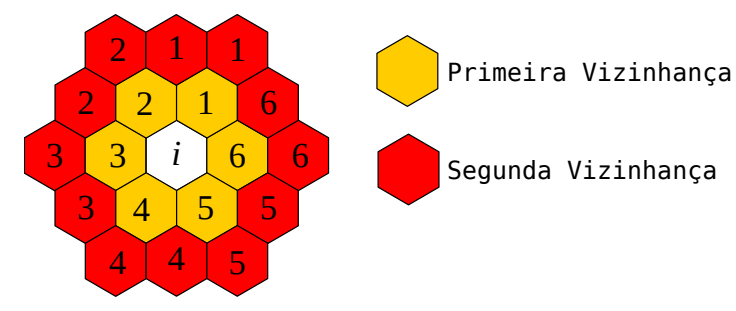

Figura 1. Vizinhanças do neurônio central $i$ e seus parâmetros angulares.

Desta forma, $d_{i}$ pode ser utilizado para indicar a distância entre 1 e 2 de forma adequada. Por exemplo, se 1 e 2 estiverem em posições diametralmente opostas em relação a $i, d_{i}$ atingirá o seu valor máximo.

\section{Metodologia Proposta para Classificação de DPs}

Neste trabalho, é proposta uma metodologia de busca pelas fronteiras de separação que maximizam a acurácia da rede SOM. Para isso, após o treinamento da rede, o conjunto de fronteiras possíveis é determinado manualmente. Compara-se a saída da rede com o rótulo de cada amostra. A metodologia seleciona o conjunto de fronteiras em que os rótulos das amostras e as saídas da rede coincidem para o maior número de amostras (maximização da acurácia).

Cada região é rotulada pela classe da maioria das amostras contidas na mesma. Todos os neurônios de uma determinada região são classificados pela rede de acordo com este rótulo. Para cada conjunto de regiões, calcula-se a taxa de acerto através de uma matriz de confusão.

$\mathrm{Na}$ Figura 2, por exemplo, observa-se um mapa com duas possíveis separações (determinadas manualmente): uma com 4 regiões e outra com 5 regiões. A matriz de 
confusão associada ao mapa com 5 regiões possui maior taxa de acerto. Neste mapa, observa-se que a área ocupada pelas amostras da classe 4 foi subdividida em duas regiões, o que indica a existência de subclasses.

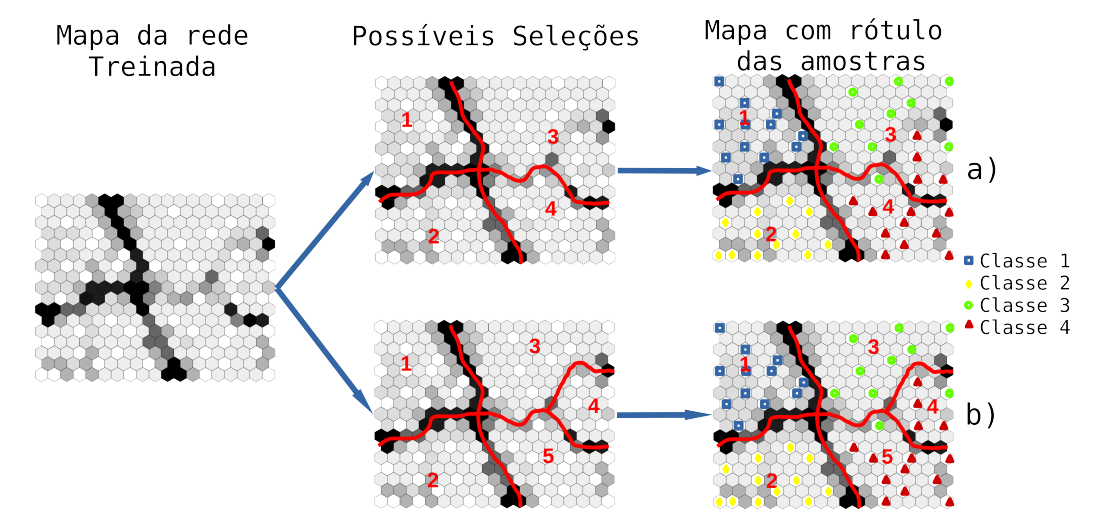

Figura 2. Processo de separação das regiões para mapas SOM.

\section{Parâmetros de Entrada}

Para a visualização dos padrões de DPs, utiliza-se o mapa estatístico $\phi-q-n$, também conhecido como PRPD (phase-resolved partial discharge) [IEC 2012]. Este relaciona a quantidade de DPs detectadas durante o tempo de aquisição em função de sua magnitude $q$ e do ângulo de fase $\phi$ da onda senoidal de $60 \mathrm{~Hz}$.

Os PRPDs da base de dados são matrizes de dimensões $256 \times 256$, totalizando 65.536 contagens de DPs. A utilização do PRPD diretamente como entrada para a rede SOM é inviável, pois o grande volume de dados demanda muitos recursos computacionais para treinamento.

Utilizou-se então a técnica de projeções de amplitude e fase do PRPD desenvolvida por [Oliveira et al. 2017]. Estas projeções conservam as informações que, segundo a norma, são essenciais para a correta identificação dos padrões de DPs, tais como o formato e simetria das nuvens e a presença ou ausência de nuvens aéreas (correspondentes aos padrões de descarga Superficial e Lacuna). Desta forma, são geradas quatro projeções, com 64 atributos cada, totalizando 256 atributos por entrada (amostra). A Figura 3 mostra um exemplo de PRPD e suas projeções correspondentes para uma amostra do tipo de Ranhura. Podem ser vistas as projeções positivas e negativas de amplitude e de fase.

\section{Resultados}

Diversas redes SOM foram treinadas, com variação da configuração da topologia, tamanho da matriz de neurônios e número total de iterações. Uma vez definidas as áreas que representam cada classe, pode-se então calcular as estatísticas de acerto e erro para o mapa. Foram utilizados $70 \%$ das amostras para treino e $30 \%$ para teste.

$\mathrm{Na}$ Figura 4(a), o mapa de Kohonen é mostrado com o algoritmo de [Han and Song 2003] (Seção 3). Já na Figura 4(b), é mostrado o mesmo mapa com separação das regiões e os rótulos das amostras que excitaram os neurônios. Esta figura valida a metodologia proposta, pois as amostras contidas no interior de cada região possuem, em 


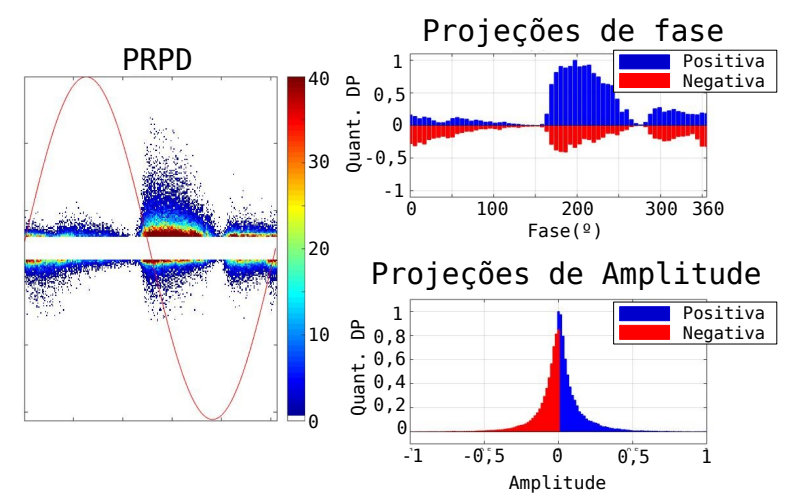

Figura 3. PRPD de Ranhura e suas projeções de amplitude e fase.

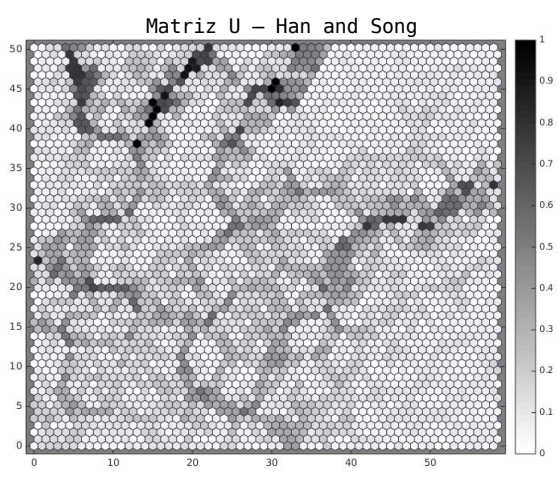

(a)

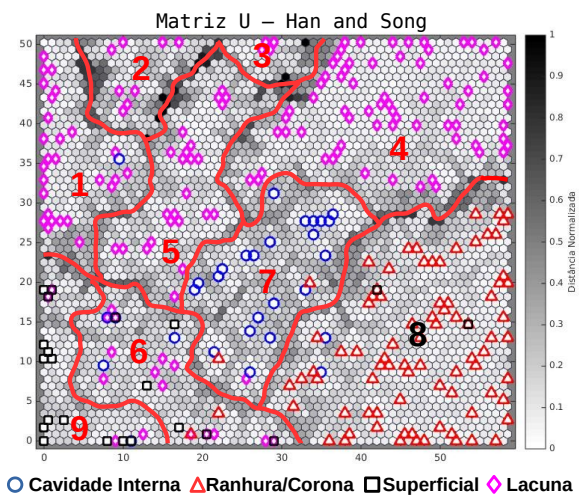

(b)

Figura 4. Visualização da rede SOM utilizando metodologia das distâncias com parâmetros angulares, a) sem marcações e b) Mostrando os possíveis agrupamentos.

Tabela 1. Matriz de Confusão para A Rede Com maiores acertos.

\begin{tabular}{|c|c|c|c|c|c|}
\hline & \multicolumn{4}{|c|}{ Classe Predita } \\
\hline & & $\begin{array}{l}\text { Cavidade } \\
\text { Interna }\end{array}$ & $\begin{array}{l}\text { Ranhura } \\
\text { /Corona }\end{array}$ & $\begin{array}{c}\text { Superfi- } \\
\text { cial }\end{array}$ & Lacuna \\
\hline \multirow{4}{*}{ 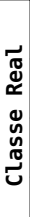 } & $\begin{array}{l}\text { Cavidade } \\
\text { Interna }\end{array}$ & $77,0 \%$ & $6,00 \%$ & $12,0 \%$ & $5,00 \%$ \\
\hline & $\begin{array}{c}\text { Ranhura/ } \\
\text { Corona }\end{array}$ & $12,5 \%$ & $84,6 \%$ & $2,90 \%$ & $0,00 \%$ \\
\hline & $\begin{array}{l}\text { Superfi- } \\
\text { cial }\end{array}$ & $6,40 \%$ & $8,80 \%$ & $75,0 \%$ & $9,80 \%$ \\
\hline & Lacuna & $1,50 \%$ & $0,00 \%$ & $18,5 \%$ & $80,0 \%$ \\
\hline
\end{tabular}

sua maioria, amostras com mesmo rótulo. Na Tabela 1, mostra-se a matriz de confusão referente a este mapa.

$\mathrm{Na}$ Figura 4(b), a rede SOM foi capaz também de identificar e separar as distintas DPs em clusters, e ainda identificou seis subgrupos de descargas de Lacuna (subgrupos enumerados de 1 a 6 ). Entende-se por um grupo uma região com considerável quantidades de neurônios excitados por amostras de um mesmo padrão. Notou-se que as DPs do tipo Lacuna, de agrupamentos diferentes, têm um comportamento de amplitude próximo (amplitude pouco variável ao longo da nuvem), mas as nuvens ocorrem em fases diferentes. 
Ressalta-se que este tipo de DP produz nuvens com intervalo de fase imprevisível e uma quantidade variável de nuvens. Assim, a metodologia de [Han and Song 2003] foi capaz de evidenciar seis sub-agrupamentos de descargas parciais, corretamente agrupando as amostras pela fase de ocorrência das nuvens, automaticamente.

\section{Conclusões}

Nesse artigo, foi apresentado o desenvolvimento de um sistema de classificação de DPs em hidrogeradores. O desenvolvimento desse sistema de classificação decorreu da necessidade de se observar a condição das máquinas, e obter indicadores para balizar o processo de manutenção. As etapas de aquisição e filtragem do sinal, combinadas com a métrica de extração de características (features), mostraram-se eficazes em obter os atributos significativos dos padrões PRPD. Isto pôde ser observado nas divisões do mapa SOM com a métrica de visualização de Han e Song.Os resultados relativos à proposta de se utilizar redes auto-organizáveis para classificar DPs de hidrogeradores foram satisfatórios, haja vista que a rede SOM, além de conseguir separar as distintas classes de DPs, automaticamente agrupou amostras do padrão Lacuna, que não apresenta, fisicamente, nuvens em faixas de fase previsíveis em cada amostra obtida durante as medições realizadas nos hidrogeradores. As amostras da classe Ranhura/Corona foram classificadas com a maior taxa de acerto (84,6\%). Já a classe Superficial teve suas amostras classificadas com o menor nível de acerto $(75,0 \%)$.

\section{Referências}

Han, Y. and Song, Y. (2003). Using improved self-organizing map for partial discharge diagnosis of large turbogenerators. IEEE Transactions on Energy Conversion, 18(3):392-399.

IEC, T. (2012). 60034-27-2: Rotating eletrical machines - part 27-2: On-line partial discharge measurements on stator winding insulation of rotating electrical machines. International Eletrotechnical Commission.

Oliveira, R., Araújo, R. C., Barros, F. J., Segundo, A. P., Zampolo, R. F., Fonseca, W., Dmitriev, V., Brasil, F. S., et al. (2017). A system based on artificial neural networks for automatic classification of hydro-generator stator windings partial discharges. Journal of Microwaves, Optoelectronics and Electromagnetic Applications, 16(3):628-645.

Stone, G. (2012). A Perspective on Online Partial Discharge Monitoring for Assessment of the Condition of Rotating Machine Stator Winding Insulation. Electrical Insulation Magazine, IEEE, 28(5):8-13.

Stone, G. C. (2005). Partial discharge diagnostics and electrical equipment insulation condition assessment. IEEE Transactions on Dielectrics and Electrical Insulation, 12(5):891-904.

Zampolo, R. F., Sena, A. J. C., Vilhena, P. R. M., Brasil, F. d. S., Dmitriev, V., and Oliveira, R. M. S. (2015). Estratégia para seleção de sub-bandas wavelet em sistemas de monitoramento de descargas parciais. XVI ERIAC, Puerto Iguazú. Al-Máquinas Elétricas Rotativas. 